\title{
Intradural lumbar disc herniation after percutaneous endoscopic lumbar discectomy: case report
}

\author{
Yasuaki Tamaki, MD, ${ }^{1}$ Toshinori Sakai, MD, PhD, ${ }^{2}$ Ryo Miyagi, MD, ${ }^{2}$ Takefumi Nakagawa, MD, PhD, ${ }^{1}$ \\ Tateaki Shimakawa, MD, PhD, ${ }^{1}$ Koichi Sairyo, MD, PhD, ${ }^{2}$ and Takashi Chikawa, MD, $\mathrm{PhD}^{1}$
}

\begin{abstract}
'Department of Orthopedics, Tokushima Municipal Hospital; and 'Department of Orthopedics, Institute of Health Biosciences, The University of Tokushima Graduate School, Tokushima, Japan
\end{abstract}

\begin{abstract}
A 64-year-old man was referred to the authors with low-back pain (LBP) and right leg pain with a history of previously diagnosed lumbar disc herniation (LDH) at L4-5. He had undergone 2 percutaneous endoscopic lumbar discectomies (PELDs) for the herniation at another institution, and according to the surgical record of the second surgery, a dural tear occurred intraoperatively but was not repaired. Postoperative conservative treatments such as an epidural block and blood patch had not relieved his persistent LBP or right leg pain. Upon referral to the authors, MRI and myelography revealed an intradural LDH. The herniated mass was removed by durotomy, and posterior lumbar interbody fusion was performed. His symptoms were partially improved after surgery. Primary suture is technically difficult when a dural tear occurs during PELD. Therefore, close attention should be paid to avoiding such tears, and surgeons should increase their awareness of intradural LDH as a possible postoperative complication of PELD.
\end{abstract}

http://thejns.org/doi/abs/10.3171/2014.12.SPINE14682

KEY WORDS intradural disc herniation; percutaneous endoscopic lumbar discectomy; complications; low-back pain

I NTRADURAL lumbar disc herniation (LDH) is an uncommon condition, first reported by Dandy in $1942 . .^{5}$ It is estimated to constitute $0.26 \%-0.30 \%$ of all disc herniations, and more than $90 \%$ of cases occur in the lumbar spine. ${ }^{2,3}$ As a possible cause of intradural LDH, some authors have suggested adhesion between the ventral dura and posterior longitudinal ligament (PLL), associated with congenital or chronic inflammation after a previous surgery. $^{2-4}$

Percutaneous endoscopic lumbar discectomy (PELD) is a recently developed minimally invasive procedure for $\mathrm{LDH}$. Although this procedure is considered to be relatively safe, complications such as dysesthesia of the leg due to exiting nerve root injury, headache, and dural tear can occur. The rate of complications for PELD is low, such as $1.9 \%$ for dysesthesia of the leg and $1.1 \%$ for dural tear., ${ }^{1,9}$ Surgical repair tends to be extremely difficult because the defects are located on the ventral side of the spine in most cases, thereby complicating surgical treatment. If the defect is not repaired, several neurological complications may occur, including extrusion of the cauda equina, symptoms related to CSF leak, and intradural LDH.
To date, no reports have described intradural LDH after PELD. In this paper we present a case of intradural LDH subsequent to a dural tear during PELD with the aim of increasing the awareness of a possible postoperative complication of PELD.

\section{Case Report}

History and Examination

A 64-year-old man presented with low-back pain (LBP) and right leg pain. He had undergone PELD for LDH at L4-5 that had been performed by another doctor. Three weeks after the initial operation, he underwent PELD again for $\mathrm{LDH}$ recurrence. A dural tear occurred during the second PELD, but suture of the defect was not performed.

Although his right leg pain was temporarily relieved after the second PELD, 2 months later he experienced right leg pain but no symptoms associated with CSF leakage, such as headache or dizziness. Magnetic resonance imaging revealed recurrent herniation of the L4-5 disc extradurally (Fig. 1). A blood patch was injected into the epidural

ABBREVIATIONS LBP = low-back pain; LDH = lumbar disc herniation; PELD = percutaneous endoscopic lumbar discectomy; PLL = posterior longitudinal ligament. SUBMITTED July 20, 2014. ACCEPTED December 12, 2014.

INCLUDE WHEN CITING Published online June 12, 2015; DOI: 10.3171/2014.12.SPINE14682.

DISCLOSURE The authors report no conflict of interest concerning the materials or methods used in this study or the findings specified in this paper. 


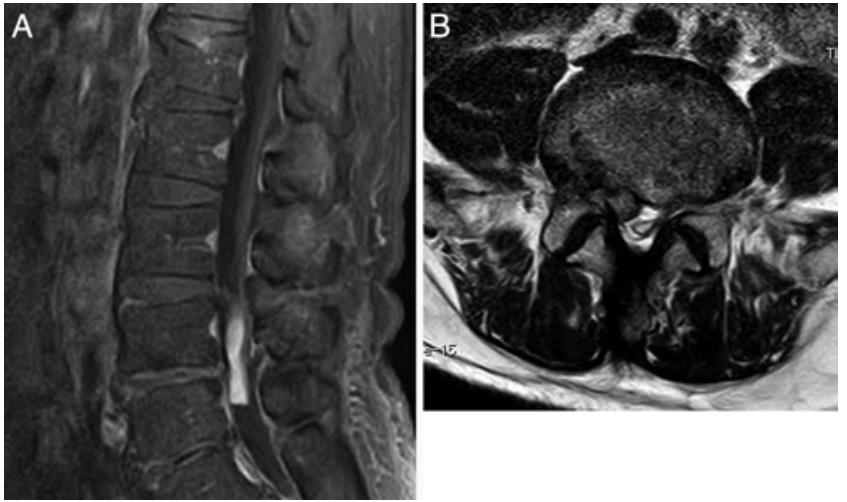

FIG. 1. Postoperative sagittal (A) and axial (B) MR images of the second PELD showing recurrence of extradural herniation at $L 4-5$.

space to sever communication between the L4-5 disc and the spinal canal. Thereafter, the patient was treated conservatively with medication and an epidural block to control his pain.

More than 2 years later LBP recurred, and the patient consulted our department for further examination. Neurological examination revealed marked right posterior thigh pain and dorsiflexion weakness of the ankle and big toe (manual muscle test score 3/5). The straight leg raising test was positive at $40^{\circ}$ on the right side. The patient also complained of dysuria.

MRI revealed an intradural mass with low intensity on T1-weighted imaging, slightly high intensity on T2-weighted imaging, and heterogeneous intensity on contrast-enhanced T1-weighted imaging (Fig. 2). It was difficult to distinguish on MRI whether the mass was located intradurally or extradurally. Computed tomography myelography clearly showed an intradural mass (Fig. 3). We judged that there was no continuity between the disc and the dural cavity. Based on the patient's clinical history and radiological findings, we strongly suspected intradural LDH.

\section{Operation and Postoperative Course}

We removed the intervertebral mass, including the herniated mass, by durotomy and interbody fusion through a posterior approach to stabilize the affected spinal level. After the durotomy, involvement of the cauda equina was observed in the intradural herniated mass (Fig. 4A). Thus, removal of the LDH with resection of adhesive scar tissue was performed. We could not see an obvious tear on the ventral dura at the time of revision surgery (Fig. 4B); the defect looked like a dimple covered by scar tissue, so we did not perform additional treatment such as suturing. There was no dorsal dural defect. We performed a primary suture to close the operative incision and covered the wound with fibrinogen glue. The postoperative pathological diagnosis was LDH with scar tissue (Fig. 5). Although neurological symptoms including muscle weakness and bladder dysfunction were improved, the patient's right leg pain persisted. At the 6-month follow-up, the manual muscle test showed improvement of muscle weakness (tibialis anterior 4/5, extensor hallucis longus 4/5). His leg pain was tolerable and he was able to walk using a walking aid.
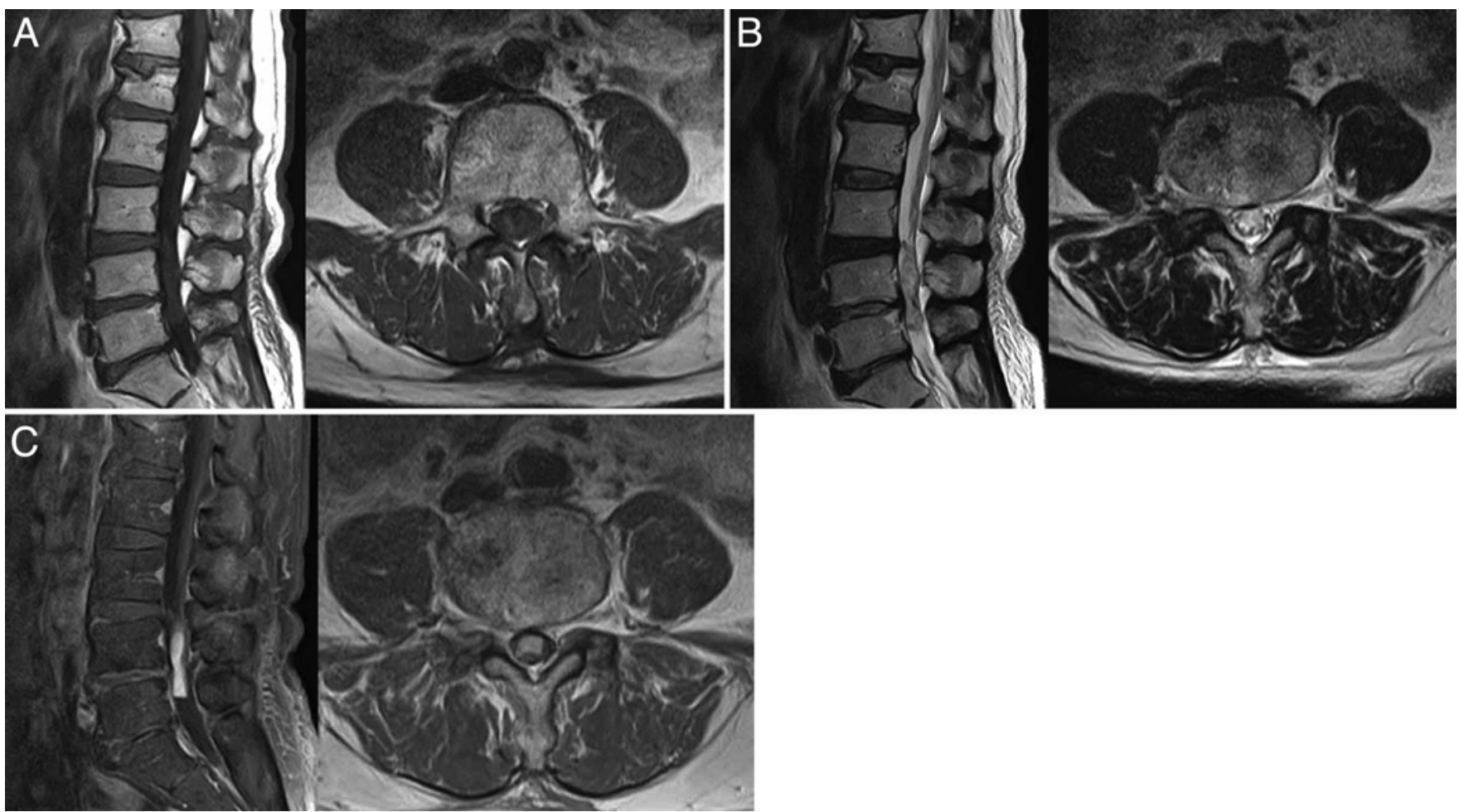

FIG. 2. Sagittal (left side) and axial (right side) MR images showing an intradural mass with low intensity on T1-weighted imaging (A), slightly high intensity on T2-weighted imaging (B), and heterogeneous intensity on contrast-enhanced T1-weighted imaging (C). 


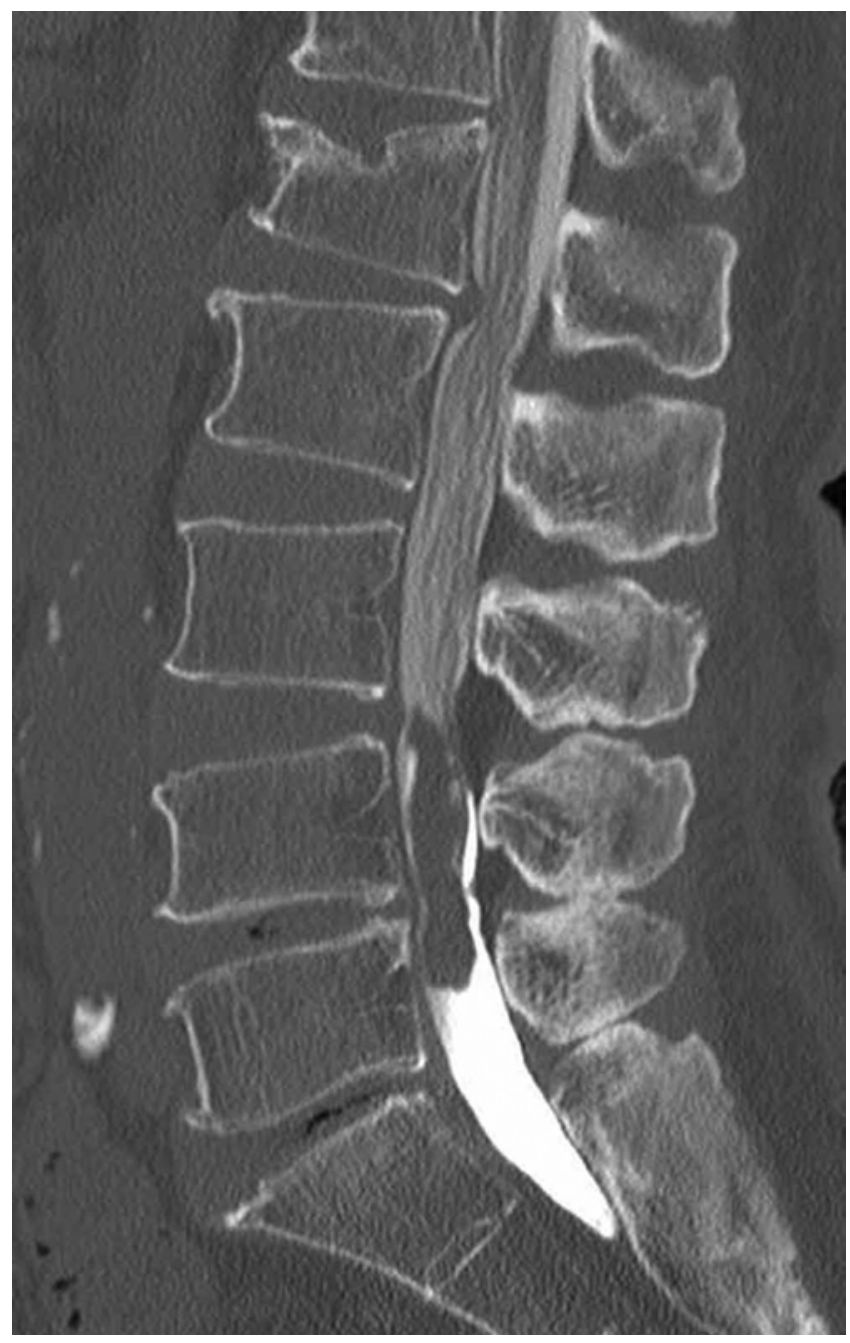

FIG. 3. Sagittal CT myelogram clearly showing an intradural mass.

\section{Discussion}

PELD is a relatively safe, minimally invasive procedure for $\mathrm{LDH}$ with a low complication rate. Although PELD is associated with specific complications, dural tearing is rare, with a reported incidence of $1.1 \% .{ }^{1}$ However, once dural tearing occurs, surgical repair is extremely difficult because the defects are located on the ventral side.

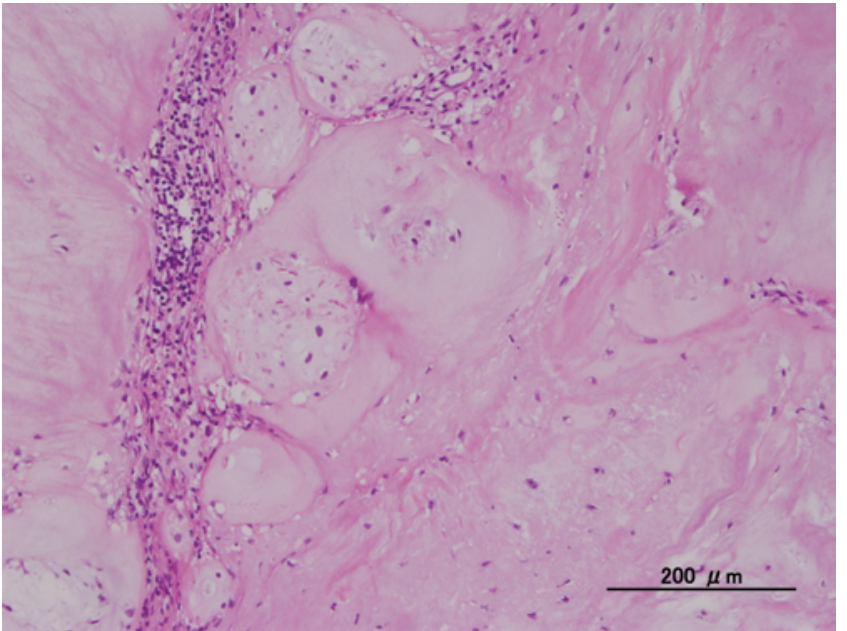

FIG. 5. The pathological diagnosis was consistent with intervertebral disc herniation and scar tissue formation. $\mathrm{H} \& \mathrm{E}$. Figure is available in color online only.

The exact pathogenesis of spontaneous intradural LDH with no previous dural injury has not been clarified, but the consensus is that chronic adhesion between the ventral dura and PLL is the main preconditioning factor of intradural LDH. ${ }^{2,3,7}$ Such adhesions may be congenital or develop after previous spinal surgery or trauma due to scar tissue formation. ${ }^{7,8}$ In the present case, intradural LDH occurred subsequent to a dural tear during PELD, which has not been previously reported.

Several radiological findings have been reported as useful tools for accurately detecting intradural LDH. Hidalgo-Ovejero et al. reported that the potential presence of intradural LDH must always be considered when performing open discectomy in cases in which CT shows the presence of epidural gas. ${ }^{8}$ Several authors have reported that MRI is the most useful imaging modality in cases of intradural LDH. Characteristic findings are the loss of PLL continuity and the "hawk-beak" sign, which shows a triangular aspect of the herniated disc compressed laterally. ${ }^{6}$ In addition, Gd contrast-enhanced MRI may show ring enhancement of the herniated portion. ${ }^{4,6}$ In the present case, MRI revealed a loss of PLL continuity but no clear hawk-beak sign or ring enhancement, and CT revealed no clear epidural gas. However, CT myelography showed obvious intradural LDH (Fig. 3).
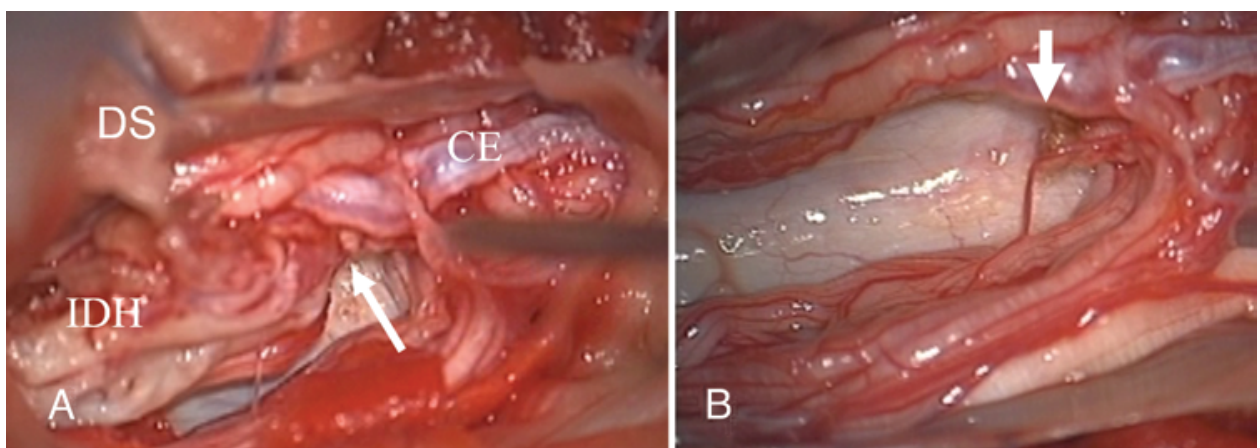

FIG. 4. Intraoperative micrographs showing involvement of the cauda equina (CE) in the intradural herniated mass. A: Adhesion was present between the intradural LDH (IDH) and ventral dura (arrow). B: After resecting the LDH, the dural defect was observed to be replaced with scar tissue (arrow). DS = dural sac. Figure is available in color online only. 
There is general agreement in previous reports that removal of the intradural LDH with durotomy and primary suture of the dural defect after decompression of the rootlets should be performed. ${ }^{2,4,7}$ However, an anterior dural tear is difficult to close by primary suture. Ducati et al. ${ }^{6}$ stated that an anterior dural tear should only be sutured when there is no risk to the surrounding rootlets. When tight closure was not absolutely necessary, they occluded the dural tear with hemostatic material without primary suture in almost all cases. ${ }^{6}$ The present case did not require a closure because the anterior dural defect had spontaneously closed.

At the time of revision surgery, we could not see an obvious tear of the ventral dura; the defect looked like a dimple covered by scar tissue. We speculate that the mechanism of the spontaneous dural seal was as follows. The epidural blood patch resulted in adhesion between the ventral dura and the disc, but it might have been fragile. The recurrent herniation could have migrated into the intradural space through the fragile portion, which was then followed by adhesion between the intradural LDH and the ventral dura. Finally, the hole was covered by scar tissue.

Primary suture is technically difficult when a dural tear occurs during PELD. Therefore, close attention should be paid to avoiding dural tears. Moreover, no optimal treatment has been established for such cases, although an epidural blood patch has been suggested. Once a dural tear occurs during PELD, surgeons should convert to open surgery; therefore, surgeons who perform PELD should be able to manage open surgery. Surgeons should be cognizant that intradural LDH is a possible postoperative complication.

\section{References}

1. Ahn Y, Lee HY, Lee SH, Lee JH: Dural tears in percutaneous endoscopic lumbar discectomy. Eur Spine J 20:58-64, 2011
2. Aydin MV, Ozel S, Sen O, Erdogan B, Yildirim T: Intradural disc mimicking: a spinal tumor lesion. Spinal Cord 42:5254, 2004

3. Choi JY, Lee WS, Sung KH: Intradural lumbar disc herniation-is it predictable preoperatively? A report of two cases. Spine J 7:111-117, 2007

4. D'Andrea G, Trillò G, Roperto R, Celli P, Orlando ER, Ferrante L: Intradural lumbar disc herniations: the role of MRI in preoperative diagnosis and review of the literature. Neurosurg Rev 27:75-82, 2004

5. Dandy WE: Serious complications of ruptured intervertebral disk. JAMA 119:474-477, 1942

6. Ducati LG, Silva MV, Brandão MM, Romero FR, Zanini MA: Intradural lumbar disc herniation: report of five cases with literature review. Eur Spine J 22 (Suppl 3):S404S408, 2013

7. Floeth F, Herdmann J: Chronic dura erosion and intradural lumbar disc herniation: CT and MR imaging and intraoperative photographs of a transdural sequestrectomy. Eur Spine J 21 (Suppl 4):S453-S457, 2012

8. Hidalgo-Ovejero AM, García-Mata S, Gozzi-Vallejo S, IzcoCabezón T, Martínez-Morentín J, Martínez-Grande M: Intradural disc herniation and epidural gas: something more than a casual association? Spine (Phila Pa 1976) 29:E463-E467, 2004

9. Yeung AT, Tsou PM: Posterolateral endoscopic excision for lumbar disc herniation: Surgical technique, outcome, and complications in 307 consecutive cases. Spine (Phila Pa 1976) 27:722-731, 2002

\section{Author Contributions}

Conception and design: Sakai, Tamaki, Chikawa. Reviewed submitted version of manuscript: Sakai, Tamaki, Miyagi, Chikawa. Study supervision: Nakagawa, Shimakawa, Sairyo, Chikawa.

\section{Correspondence}

Toshinori Sakai, Department of Orthopedics, Institute of Health Biosciences, The University of Tokushima Graduate School, 3-18-15 Kuramoto-cho, Tokushima 770-8503, Japan. email: norinori@tokushima-u.ac.jp. 